\title{
Erratum: Local correlated motions in aqueous solution of sodium chloride [Phys. Rev. Materials 3, 065604 (2019)]
}

\author{
Yuya Shinohara $\oplus^{\circ}$, Wojciech Dmowski, Takuya Iwashita, Daisuke Ishikawa, \\ Alfred Q. R. Baron, and Takeshi Egami
}

(Received 29 August 2019; published 4 October 2019)

DOI: 10.1103/PhysRevMaterials.3.109902

In the original paper, we inadvertently used an incorrect time axis for the figures and fitting procedures. It was too large by a factor of 2. All the values of time need to be the half of those in the original paper. This influences the timescales in Figs. 2, 4, 6, and 7 and the fitted parameter of $\tau_{1}$ and $\tau_{2}$ in Figs. 5 and 6; all the time axes are reduced by a half. The necessary corrections are as follows:

(1) The text on p. 4, left column, last paragraph should be changed to: "At a longer timescale $[t>0.25$ (rather than 0.5 in the original paper) ps], the addition of salt induces a qualitative change at the first- and the second-neighbor peaks." and "Therefore, the first-neighbor correlation is dominant in the $\mathrm{NaCl}$ aqueous solution at $t>0.5 \mathrm{ps}$." In addition to the changes in the timescale, we reexamined the energy resolution function. The correct values for $\tau_{G}$ is $0.1 \mathrm{ps}$ and for $\tau_{E}$ is 1 ps on p. 2 .

(2) The text on p. 6, left column, last paragraph of Sec. III B. should be changed to: "The fact that all the decay curves intersect at $t=0.14$ ps supports that the spectra are decomposed into the linear combination." The text on p. 6, left column, the paragraph next to Eq. (10) should be changed to "At a short time ( $t<0.3 \mathrm{ps})$, positive correlation peaks are observed ... ." The text on p. 6, right column, last paragraph should be changed to: "due to a contamination of termination errors in the Van Hove functions at a short time, particularly at $t<0.1 \mathrm{ps},[\cdots]$ approximated by a two-step relaxation, $I(t)=1.5 \exp \left[-\left(\frac{t}{0.16 \mathrm{ps}}\right)^{1.7}\right]=$ $0.6 \exp (-t / 1.0 \mathrm{ps})$ as shown by the solid line in Fig. 7 (bottom). The first term is almost identical to that of pure water $\left(\tau_{1}=0.16\right.$ ps), except for its amplitude, indicating that the decaying at a short timescale $(t<0.15 \mathrm{ps})$ corresponds to a ballistic, [ $\cdots]$."

(3) The text on p. 7, left column, first paragraph should be changed to "that of water-water correlations with a decaying time $\sim 1$ ps," The text on p. 7, left column, last paragraph should be changed to "The values of the normalized amplitude remain almost constant for $t<0.15 \mathrm{ps}$.

(4) Timescales of Figs. 2, 4, 6, and 7 should be changed, and the fitting result in Fig. 5 was updated. The complete Figs. 2, 4, 5,6 , and 7 are reprinted here.

The discussion and the conclusions presented in our original paper remain unaffected.
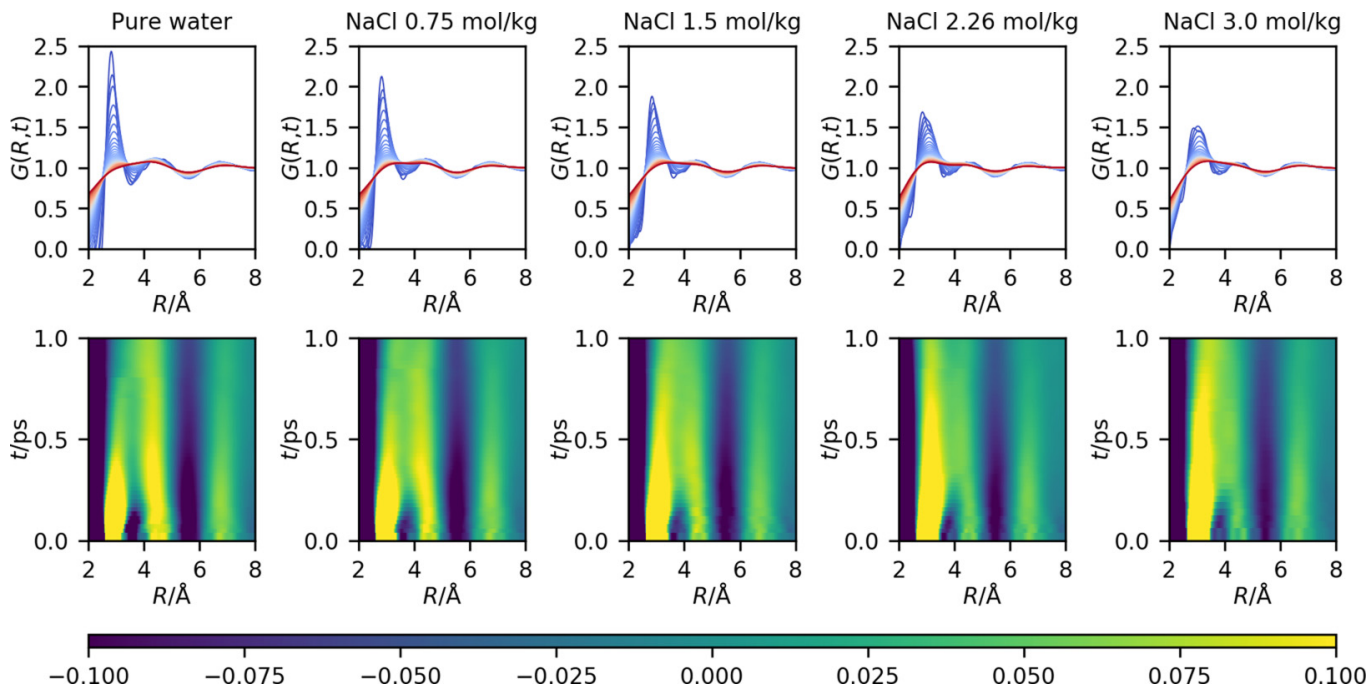

FIG. 2. The Van Hove function of a $\mathrm{NaCl}$ aqueous solution. (Top) One-dimensional slice of the Van Hove functions $G(R, t)$ at $0<t<1$ ps. (Middle) Intensity map of $G(R, t)-1$, the color scale of which is shown on the bottom. Left to right: $m=0$ (pure water), $0.75,1.5,2.26$, and $3.0 \mathrm{~mol} / \mathrm{kg}$. 


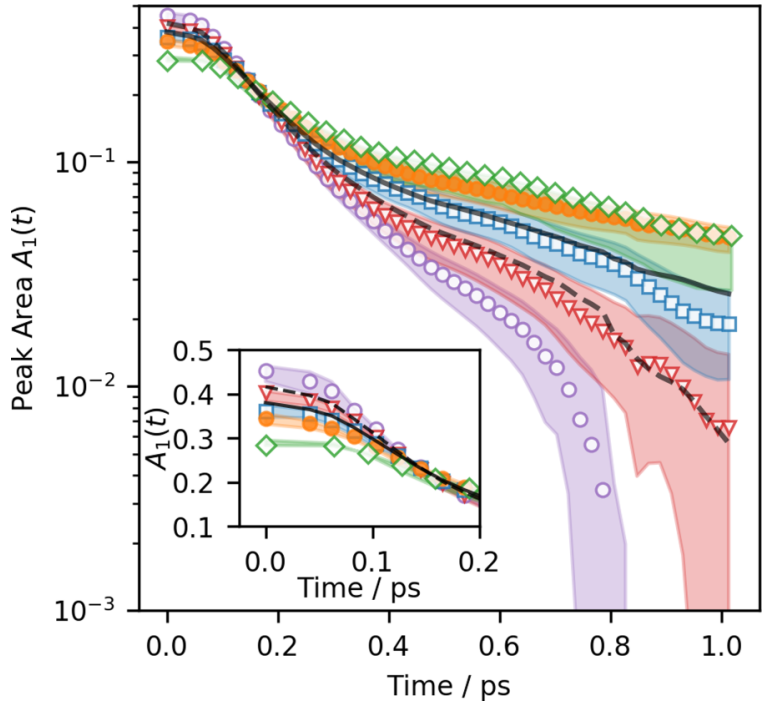

FIG. 4. Temporal changes in the area of the first-neighbor peak, $A_{1}(t)$, and the enlarged view (inset): (open circles) pure water, (triangles) $m=0.75 \mathrm{~mol} / \mathrm{kg}$, (squares) $1.5 \mathrm{~mol} / \mathrm{kg}$, (closed circles) $2.26 \mathrm{~mol} / \mathrm{kg}$, and (diamonds) $3.0 \mathrm{~mol} / \mathrm{kg}$. The shaded areas represent uncertainties of each dataset. The solid and dashed lines represent the linear combination of time evolution for $m=0$ and $2.26 \mathrm{~mol} / \mathrm{kg}$ (see the main text).
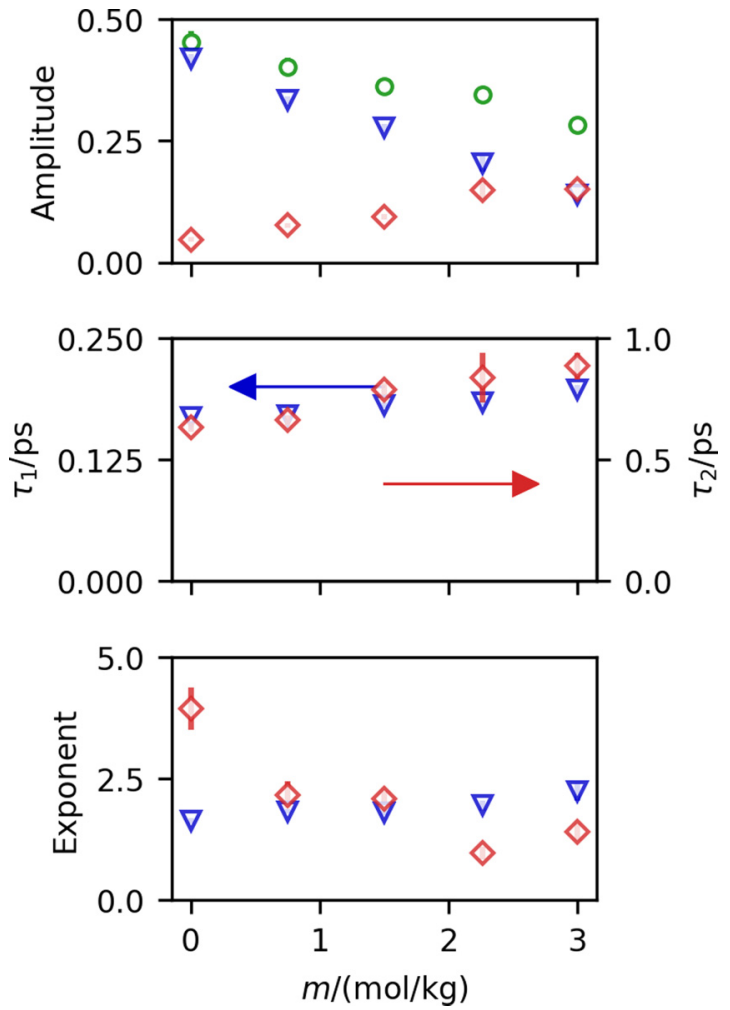

FIG. 5. Fitting results of the first-neighbor decay. (Triangles) $a_{1}$, $\tau_{1}, p_{1}$, (diamonds) $a_{2}, \tau_{2}, p_{2}$, and (circles) $A_{1}(0)$. The error bar is estimated by the standard deviation of the fitting.
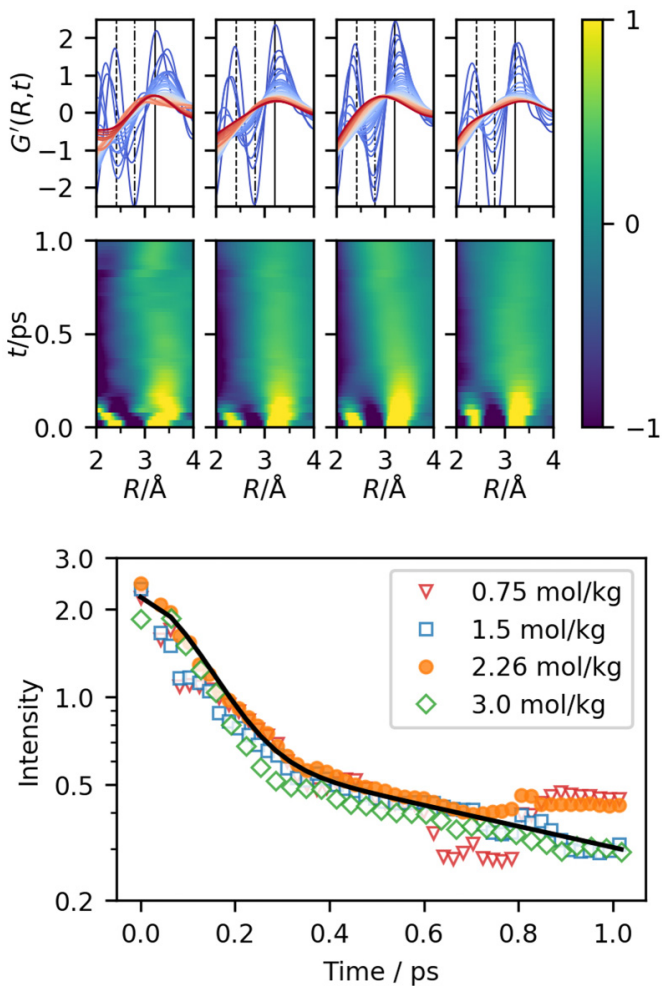

FIG. 6. (Top) One-dimensional profiles of $G^{\prime}(R, t)$ at $0<t<$ $1 \mathrm{ps}$ and (middle) their intensity maps. Their color scale is shown on the right. The molality of the sample is $0.75,1.5,2.26$, and $3.0 \mathrm{~mol} / \mathrm{kg}$ from the left to the right. The solid lines, dashed lines, and the dashed-dotted lines in the top figures represent $R=3.21 \AA\left(R_{\mathrm{O}^{2-}}+R_{\mathrm{Cl}^{-}}\right), R=2.42 \AA\left(R_{\mathrm{O}^{2-}}+R_{\mathrm{Na}^{+}}\right)$, and $R=2.8 \AA\left(R_{\mathrm{O}^{2-}}+R_{\mathrm{O}^{2-}}\right)$, respectively. (Bottom) Time evolution of the peak height at around $R=3.21 \AA$. The solid line shows the result of fitting using two (compressed) exponential functions (see the main text)

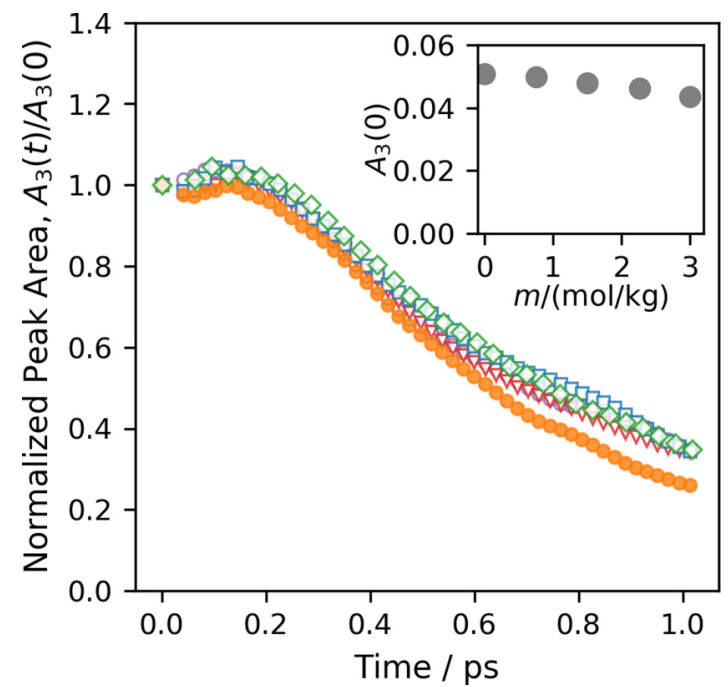

FIG. 7. Normalized temporal changes in the area of the third peak, $A_{3}(t) / A_{3}(0)$, around $R=6.8 \AA$; (open circles) pure water, (triangles) $m=0.75 \mathrm{~mol} / \mathrm{kg}$, (squares) $1.5 \mathrm{~mol} / \mathrm{kg}$, (closed circles) $2.26 \mathrm{~mol} / \mathrm{kg}$, and (diamonds) $3.0 \mathrm{~mol} / \mathrm{kg}$. Inset: Dependence of $A_{3}(0)$ on the molality. 